Michael Lee, Maria J. Lopez-Martinez, Abdoullatif Baraket, Nadia Zine1 Jaume Esteve, Jose A. Plaza, Naveed Ahmed, Abdelhamid Elaissari, Nicole Jaffrezic-Renault and Abdelhamid Errachid,

"Combination of PDMS microfilters and micromixers based on flexible thermoplastic films for size sorting and mixing of microparticles",

J. Appl. Polym. Sci., 132, 2015

doi: 10.1002/app.42088

https://onlinelibrary.wiley.com/doi/abs/10.1002/app.42088 


\section{Combination of PDMS microfilters and micromixers based on flexible thermoplastic films for size}

sorting and mixing of microparticles

Michael Lee, ${ }^{1}$ Maria J. Lopez-Martinez, ${ }^{2 *}$ Abdoullatif Baraket, ${ }^{1}$ Nadia Zine, ${ }^{1}$ Jaume Esteve, ${ }^{2}$

Jose A. Plaza, ${ }^{2}$ Naveed Ahmed, ${ }^{3 \dagger}$ Abdelhamid Elaissari, ${ }^{3}$ Nicole Jaffrezic-Renault, ${ }^{1}$

Abdelhamid Errachid ${ }^{1}$

ABSTRACT: We present a study for the development of flexible microfilters based on sealing microstructured poly(dimethylsiloxane) (PDMS) to different functionalized thermoplastic films [polyimide (PI), polyethylene naphthalate (PEN), and polyethylene terephthalate (PET)]. The microfilter was manufactured by soft-lithography and replica molding and then combined with plasma activation and chemical treatment using 3-(aminopropyl)triethoxysilane (APTES). To demonstrate the functionality of the PDMS microfilters, poly(lactic-co-glycolic acid) (PLGA) microparticles (MPS) were filtered through the microfluidic device based on the three thermoplastic films. Subsequently, the mixing capabilities of a passive PDMS micromixer was observed with the injection of polymeric MPS (fluorescent and nonfluorescent) as fluidic mixers are not generally effective at mixing particles. On mixing nonfluorescent MPS (<10-30 $\mathrm{mm}$ in diameter) a mixing performance of $13.3 \%$ at $5 \mathrm{~mm}$ was observed. Therefore, a PDMS microfiltering device was integrated with a PDMS micromixer using a simple and cost effective home-made polymeric connector for filtration at a size sorting of $11 \mathrm{~mm}$. The results exhibit that the combination of the two microfluidic devices can be achieved with size sorting and mixing of MPS with an improved mixing performance of $62.5 \%$ at 3 mm. VC 2015 Wiley Periodicals, Inc. J. Appl. Polym. Sci. 2015, 132, 42088. 
KEYWORDS: applications; elastomers; functionalization of polymers; microfluidics; thermoplastics

\section{INTRODUCTION}

In the microfluidics community, poly(dimethylsiloxane) (PDMS) as a prototyping material has been sealed between PDMS and PDMS, or other materials such as glass and siliconbased wafers. ${ }^{1-4}$ However, there has been a recent interest in developing new polymer materials as substrates. ${ }^{5-7}$ These techniques provide robust and rigid structures to be developed into hard thermoplastic films that have relatively low costs, flexibility, biocompatibility, and display advantageous electrical, physical, mechanical, and thermal properties. ${ }^{8}$ However, to process these materials with microfluidic structures, expensive embossing machines and fabricated positive master molds are required in a clean room environment. As the optical characteristics of the polymers can rival those of glass, ${ }^{9}$ interest in polymers as substrates for microfluidics has led to an increase in literature demonstrating the feasibility of thermoplastic films as a material to enclose PDMS microfluidic devices. ${ }^{10-14}$ Though PDMS is inherently a soft material, production of molds in the microand nanometer resolution are cheap, quick to produce, ${ }^{15}$ and do not require specialized or sophisticated machinery and laboratories. Therefore, high quality and complex structures can be replicated into PDMS that are durable and conformable to a

nonplanar surface within the micrometer scale. ${ }^{16,17}$ The diverse material properties of PDMS and thermoplastic films, therefore, can provide unique opportunities to fabricate hybrid microfluidic devices. ${ }^{14}$ To seal the developed PDMS microfluidic device, thermoplastic films as a substrate requires surface functionalization with 3-(aminopropyl)triethoxysilane (APTES). Li and Wilkes have reported a method for covalently bonding APTES containing solgel coatings to modified polycarbonate (PC) substrates. ${ }^{18}$ The authors demonstrate the applicability of APTES to generate amide bonds on the polymer surface. In an early article by $\mathrm{Hu}$ et al., ${ }^{19}$ the authors incubated polyethylene naphthalate (PEN) films (without plasma 
treatment) in APTES dissolved in toluene for $24 \mathrm{~h}$. The substrates were then hydrolyzed in distilled water over-night to induce $\mathrm{Si}(\mathrm{OH})_{3}$ groups. More recently, Tang and Lee have activated poly(methylmethacrylate) (PMMA), PC, polyimide (PI), and polyethylene terephthalate (PET) films with an epoxy-silane and created a covalent bond with an amino-silane functionality based on PDMS. ${ }^{12}$ Large assemblies [e.g., a serpentine microchannel $(186 \mathrm{~mm} 3500 \mathrm{~mm} 3150 \mathrm{~mm})$ ] were examined for leakage. Finally, Sunkara et al. have bonded PDMS [serpentine microchannel (70 mm $3800 \mathrm{~mm} 345 \mathrm{~mm}$ )] to PC, cyclic olefin copolymer (COC), PMMA, and polystyrene (PS) films through APTES activation. ${ }^{13}$ These authors have demonstrated that PDMS bonded to thermoplastic films through APTES bonding can withstand the pressure requirements necessary for microfluidic applications. Different films can provide varying surface environments (surface, optical, and mechanical properties) and, therefore, the films can be chosen for a specific application that can be influenced to the largest extent. ${ }^{20}$

Until recently, the potential applications for APTES bonding have not been specifically reported. In the literature, there are many reported PDMS microfilters using polymer substrates, typically where filtration is based upon cross-flow filtration through a porous polymer membrane. For instance, Gu and Miki applied two PDMS microchambers sandwiching a porous membrane of polyethersulfone (PES) by the wet-phase inversion method. ${ }^{21}$ The PES membrane functioned as a barrier to sodium chloride $(\mathrm{NaCl})$ and fluorescein isothiocyanate (FITC) dextrans sized between 0.3 and $6.6 \mathrm{~nm}$. Aran et al. applied two PDMS layers to sandwich a porous PC (PCTE) with activation of the membrane by APTES $(5 \% \mathrm{v} / \mathrm{v})$. The application of filtering whole blood plasma was infused and the porous membrane (200 $\mathrm{nm}$ pore size) was unaffected by the activation with APTES. ${ }^{11}$ Recently, we have reported on the fabrication of PDMS passive micromixers that were bonded to PI, PEN, and PET films using an aqueous solution of APTES $(6 \% \mathrm{v} / \mathrm{v}) .{ }^{22}$ The investigation of mixing water-based solutions demonstrated that mixing liquids was successful through chaotic advection and recirculation; however, in real world applications micromixers are needed to mix suspensions or particles. For instance, Choi et al. created a microfluidic and electrochemical detection 
device that incorporated microvalves, flow sensors, biofilters, and immunosensors. ${ }^{23}$ Bulk micromachining and wafer bonding techniques patterned the applied glass wafer and electrostatically bonded it to a silicon wafer. The particle bead-based immunoassay technique coated antibodies was held at the biofilter by the application of a magnetic field. The biofilter was designed to retain and separate magnetic beads from a fluidic suspension. Target antigens were then injected and separated due to the specific antibody/antigen interaction. Yuen et al. produced a glasssilicon microchip that contained flow deflectors and weir-filters for mixing and filtration of human whole blood for the isolation of white blood cells. ${ }^{24}$ Finally, Bhagat and Papautsky observed the effectiveness of conventional PDMS micromixers for mixing particle flows on plasma bonded glass slides. ${ }^{25}$ To enhance particle mixing, the polystyrene particles required lateral movement within the microchannels and obstructions were introduced to increase mixing. A Y-mixer, Telsa, and obstruction mixers were analyzed respectively with the obstruction micromixer being the most favored for achieving particle dispersion when using a wide range of particle sizes. The authors noted that both Telsa and obstruction mixers achieved $90 \%$ fluid mixing in $5 \mathrm{~mm}$. However, with particle dispersion the modified Telsa design was $60 \%$ effective at $3 \mathrm{~mm}$, while the obstruction mixer was $90 \%$ at $3 \mathrm{~mm} .{ }^{25}$ These last few examples, demonstrate filtering and mixing based on either two hydrophilic surfaces (silicon-glass) or one hydrophilic and the other hydrophobic (PDMS). Therefore, the aim of this article is to combine these two microfluidic devices together (microfilter and micromixer) and to observe the possibilities of filtering and mixing varying particle sizes in relatively hydrophobic materials. Applying the filter connected to the mixer, we observed the efficiency of the mixer's capability to uniformly mix the particles over a short microchannel length with a transverse velocity component (18 mixing loops).

In this article, flexible nonporous polymer substrates of PI, PEN, and PET were functionalized with APTES. Afterwards, the films were bonded onto oxygen activated PDMS microfiltering and micromixer devices. The feasibility of filtering using pillar filters was monitored with poly(lactic-co-glycolic acid) 
(PLGA) microparticles (MPS). Afterwards, the mixing capabilities of the micromixer with MPS in poly(vinyl alcohol) (MPS-PVA) and fluorescent labeled MPS with Nile red (MPS-NR) was observed. Finally, the PDMS microfilter was connected to the PDMS micromixer for filtration of MPS with dimensions of $<11 \mathrm{~mm}$. Those particles which were smaller then entered into the mixer and the mixing performance was observed. To our knowledge, we have exhibited the first complex PDMS microfilter and micromixer that has been combined for particle size sorting and mixing based on the aforesaid thermoplastic films. These results display the development of microfluidic components using APTES for PDMS-thermoplastic film bonding that is based on a simple connection for lab-on-a-chip (LoC) devices where real samples involve filtering and mixing of particles or suspensions. This is driven by the requirement to perform absolute separation of the MPS from the microscale fluidic volume, while rapid mixing is crucial for reactions that require mixing of the reactants over a short microchannel length. In this instance, this article provides the preliminary results of microfiltering and micromixing of MPS with the future aim of size sorting whole blood samples where red blood cells will be microfiltered and the plasma cells will then be mixed with reagents for LoC-based biosensors.

\section{EXPERIMENTAL}

Materials and Techniques

Chemicals. All chemicals were purchased from Sigma-Aldrich, France apart from toluidine blue (TB) (Carl Roth GmbH \& Co, France), iron (III) nitrate nonahydrate from Acros Organics, France, dichloromethane (DCM) (Carlo Erba Reagents, France), and Nile red (NR) from Molecular Probes, Leiden, The

Netherlands. 

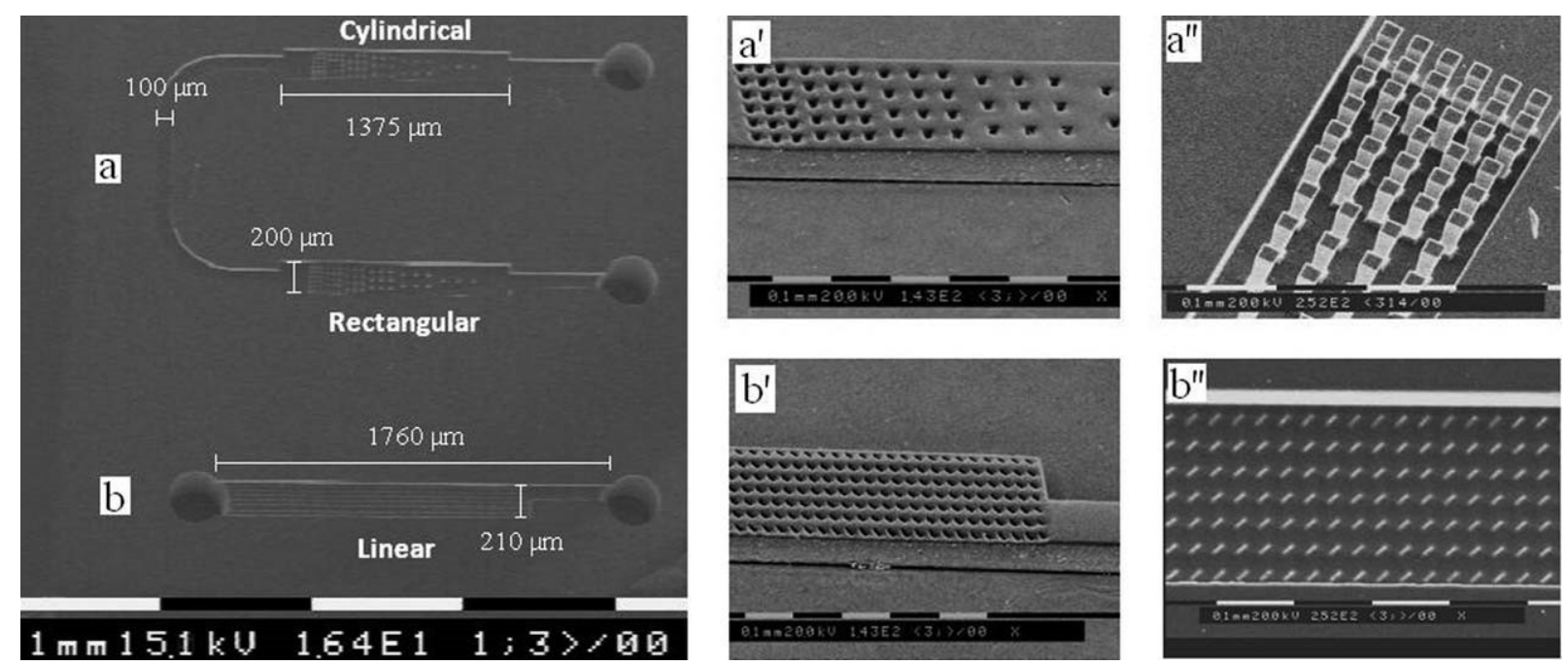

Figure 1. SEM images of the overall dimensions of the silicon master with microfilters based on (a) ' $U$ ' shaped, and (b) linear with: $\left(a^{0} / b^{0}\right)$ the first

PDMS replica at a ratio of $5: 1(\mathrm{w} / \mathrm{w})$, producing a positive microfluidic channel with negative features, and $\left(\mathrm{a}^{00} / \mathrm{b}^{00}\right)$ the final mold made from cured PDMS at a ratio of $10: 1(\mathrm{w} / \mathrm{w})$.

Polymers. PI (HN $125 \mathrm{~mm}$ ) was purchased from DuPont, and poly(ethylene naphthalate) (PEN, $160 \mathrm{~mm}$ ) and poly(ethylene terephthalate) (PET, $200 \mathrm{~mm}$ ) were purchased from Goodfellow. All polymers were used as supplied. PDMS (Sylgard 184) was purchased from Dow Corning, France. Poly(lactic-co-glycolic acid) (PLGA) MPS (RESOMER RG 502H) with a copolymer lactide-glycolide ratio of 48 : 52-52:48 was supplied by Boehringer Ingelheim (Germany) and poly(vinyl alcohol) (PVA, MowiolVR 4-88. $\mathrm{M}_{\mathrm{w}} 531,000 \mathrm{~g} / \mathrm{mol}$ ) (Sigma Aldrich, Germany). Poly(ethyl acrylate-co-methyl methacrylate-cotrimethylammonioethyl methacrylate chloride) 1: 2: 0.1 Eudragit RS-100 was purchased from EVONIKVR, Germany.

Optical Imaging. All optical images were taken by optical microscopy (Olympus BX41M, France and Leica EZ4D, Spain) and a fluorescence microscope (Zeiss Axio Scope.A1, France). The software Stream 
Start from OlympusVC was employed for characterization of the filtering dimensions and diameters of the MPS. Scanning electron microscope (SEM) images were taken with a SEM 515 (Philips, Holland).

\section{Microfilter Designs}

The silicon microfilter fabrication technology is the same as previously reported for the fabrication of microdispensers. ${ }^{26}$ The versatility of this fabrication technology is demonstrated, however, as rather than transferring micro-dispenser patterns during the photolithographic step, microfilter shapes were patterned on the top side of the wafer.

A ' $U$ ' shaped microfluidic channel containing two filtering systems that run parallel to one another was etched in 4 inch $500 \mathrm{~mm}$-thick silicon with cylindrical and rectangular pillar structures [Figure 1(a)]. Cylindrical and rectangular structures were designed on the same channel to test deep reactive ion etching (DRIE) separately with structures that can be fabricated for a pretreatment on-chip process. ${ }^{27}$ For the introduction of a sample solution into the injection port, the liquid enters into the filtering system with the widest spaced filtration structures (Set 1). After flowing through either the circular or rectangular structures, the liquid encounters the second filtering system (Set 2) with closer spaced structures, and so on. The dimensions of the filtering structures, measured across the x-axis, are as follows: Set 1, $90 \mathrm{~mm}$; Set 2, $53 \mathrm{~mm}$; Set 3, $34 \mathrm{~mm}$; Set 4, $23 \mathrm{~mm}$; Set 5, $15 \mathrm{~mm}$; and Set 6, $11 \mathrm{~mm}$. The total microchannel length and width were fabricated to $1375 \mathrm{~mm}$ and $200 \mathrm{~mm}$, respectively. The heights of the PDMS pillars within the microfluidic channel were $75 \mathrm{~mm}$.

A third filtering system was included in a linear channel and consisted of angled rectangular $2137 \mathrm{~mm}^{2}$ structures [Figure 1(b)] spaced by a distance of $14 \mathrm{~mm}$ between columns (y-axis) and $10 \mathrm{~mm}$ between rows (x-axis). The channel length and width were $1760 \mathrm{~mm}$ and $210 \mathrm{~mm}$, respectively. The total surface area of the microfilter device was $24 \mathrm{~mm}^{2}$. 
PDMS Replica Molding

The original silicon microfilters were anodically bonded to sand-blasted glass wafers. ${ }^{27}$ In this article, we have applied the silicon microfilter device as a master mold. Therefore, after DRIE the structured silicon master was cleaned and activated in freshly prepared piranha solution $\left(\mathrm{H}_{2} \mathrm{SO}_{4}: \mathrm{H}_{2} \mathrm{O}_{2} 23: 1\right.$, v/v) for $10 \mathrm{~min}$. The structured master was then silanized with octatrichlorosilane (OTS) to permit demolding of the PDMS replica. ${ }^{28}$

The silicon mold was fabricated with positive features within a negative microfluidic channel [Figure $1(a, b)]$ and, therefore, two PDMS moldings were necessary to fabricate an exact replica. From the structured silicon master, an elastomeric replica was fabricated from PDMS at a ratio of $5: 1$ (elastomer: crosslinking agent, w/w) by soft-lithography [Figure $\left.1\left(\mathrm{a}^{0}, \mathrm{~b}^{0}\right)\right]$. The PDMS was cured at 90C for $1 \mathrm{~h}$ and then removed from the silanized silicon.

This first PDMS replica mold was then coated with a thin-layer of gold $(6.25 \mathrm{~nm})$ using an Emscope SB 500 Sputter Coater

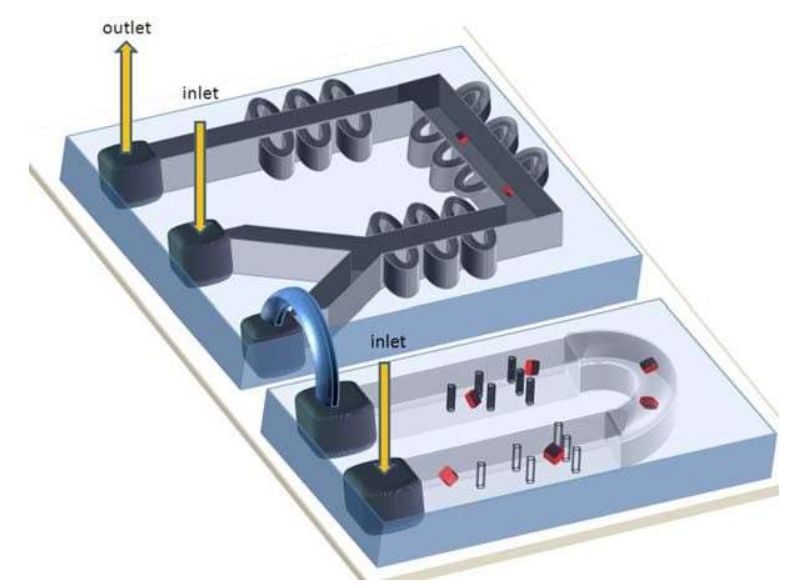

Figure 2. Schematic representation of the PDMS microfilter and micromixer bonded onto the same thermoplastic film for the integration of the two devices for size sorting followed by mixing. [Color figure can be viewed in the online issue, which is available at wileyonlinelibrary.com.] 
(France) for 30s (21 Torr). ${ }^{29}$ The gold layer acts as an antiadhesive layer that will enable the final PDMS replica to be removed from the first PDMS replica after curing. A standard PDMS elastomer/cross-linker mixture with a ratio of $10: 1(\mathrm{w} / \mathrm{w})$ was poured into the first PDMS mold. This created a replica that was an exact copy of the original silicon master with the required microfluidic channels [Figure $1\left(\mathrm{a}^{00} / \mathrm{b}^{00}\right)$ ]. This second replica was also cured at 90C for $1 \mathrm{~h}$.

PDMS and Polymer Functionalization and Adhesion

The polymers were cleaned by sonication in propanol and then Milli-Q for 10 min, respectively. Afterwards, the films were thoroughly rinsed in Milli-Q and dried with nitrogen. The polymer films were then activated by oxygen plasma (Plasma Technology, USA) for $1 \mathrm{~min}(90 \mathrm{~W}, 100 \mathrm{mTorr})$. This ensured that surface carbonyl groups were activated on the thermoplastic films, making the surfaces highly hydrophilic for the incubation in APTES solution $(6 \%, v / v)$. Incubation was maintained for 20 min at 90C Finally, the substrates were rinsed in Milli-Q, dried with nitrogen, and immediately used.

The PDMS microfilter replicas were sonicated in ethanol for $10 \mathrm{~min}$, rinsed in Milli-Q, and dried with nitrogen. Afterwards, the PDMS replicas were activated with oxygen plasma for 20 s (90 W, 100 mTorr) and positioned channel-side down onto the APTES-modified surface of the polymer films. Applied pressure to the PDMS device ensured that a conformal contact with the polymer surface (PI, PEN, and PET) was maintained. Finally, the completed microfluidic devices were placed in the oven at 90C for $1 \mathrm{~h}$ to allow silanization to occur between the structured PDMS and the polymer film.

For the application and integration with the PDMS micromixer, the same techniques (sections PDMS Replica Molding and PDMS and Polymer Functionalization and Adhesion) were applied. Specifications of the micromixer can be found in Ref. 22. The two microfluidic devices were bonded onto the same PET film. After APTES bonding to the thermoplastic film, PC tubing (internal and external diameter of 0.25 and 
$0.76 \mathrm{~mm}$, respectively) were cut to $10 \mathrm{~mm}$. Syringe tips (Robocol, France) with an external diameter of $0.31 \mathrm{~mm}$ and an internal diameter of $0.16 \mathrm{~mm}$ were inserted into the PC tubing and severed. The tube with tips was then connected to the outlet of the ' $U$ ' shaped PDMS microfilter (cylindrical structures) and into the closest Y-shaped PDMS micromixer inlet. The principle of the microfluidic system is shown in Figure 2.

Preparation of Microparticles

The MPS-PLGA was prepared using a modified double-emulsion method. ${ }^{30,31}$ The water-in-oil (W/O) emulsion was created using $250 \mathrm{~mL}$ of water that was poured into the organic phase (DCM). The organic phase was made by dissolving $500 \mathrm{mg}$ of PLGA in DCM $(2 \mathrm{~mL})$. After pouring the aqueous phase into the organic phase, the stirring was controlled using a high shear mixer/emulsifier (T-25 ultra-turrax, IKAworks, USA) at 13,000 rpm for $1 \mathrm{~min}$. This first emulsion $\left(\mathrm{W}_{1} / \mathrm{O}\right)$ was then rapidly transferred to the aqueous phase (a mixture of $2.5 \mathrm{~mL}, 2 \%$ PVA, and $47.5 \mathrm{~mL}$ distilled water that resulted in a $50 \mathrm{~mL} 0.1 \%$ PVA solution) while stirring at $6500 \mathrm{rpm}$ for $1 \mathrm{~min}$. The double emulsion that was produced (water-inoil-in-water: $\left.\mathrm{W}_{1} / \mathrm{O} / \mathrm{W}_{2}\right)$ was then transferred to a large volume of water $(100 \mathrm{~mL})$ under mechanical stirring for $2.5 \mathrm{~h}$ until all the organic solvent was extracted to the external solvent. The produced MPSPLGA were characterized at $<10-50 \mathrm{~mm}$ in diameter.

Two other types of polymeric MPS were prepared using cationic Eudragit RS-100 and the same process as described above was followed by the solvent evaporation step. Here, $1 \mathrm{~mL}$ of $0.5 \%$ PVA aqueous solution at $0.5 \%(\mathrm{w} / \mathrm{w})$ was first emulsified for 2 min by Ultraturrax at 13,500 rpm into the polymer solution $\left(1 \mathrm{~g}\right.$ of polymer) in DCM $(5 \mathrm{~mL})$. This first emulsion $\left(\mathrm{W}_{1} / \mathrm{O}\right)$ was then poured into $50 \mathrm{~mL}$ of PVA solution at $0.5 \%(\mathrm{w} / \mathrm{w}) . \mathrm{A} \mathrm{W}_{1} / \mathrm{O} / \mathrm{W}_{2}$-emulsion was formed by a second emulsification at $13,500 \mathrm{rpm}$ for $2 \mathrm{~min}$. Finally, the $\mathrm{W}_{1} / \mathrm{O} / \mathrm{W}_{2}$ emulsion was diluted in $100 \mathrm{~mL}$ of PVA solution at $0.5 \% \mathrm{w} / \mathrm{w}$ by magnetic stirring at $500 \mathrm{rpm}$ and dried overnight under the extractor hood. This produced the first MPS-PVA. 
For the second type of particles, MPS were fluorescently labeled with Nile Red (NR). Here, the particles were prepared according to the protocol described previously; however, before preparing the W/O emulsion, NR was dissolved in the organic phase (DCM) containing Eudragit RS-100. As NR has a strong susceptibility to fluorescence bleaching, only a small quantity (a few mg) of the powder was used. Both MPS-PVA and MPS-NR were $<10-30 \mathrm{~mm}$ in diameter.

\section{Characterization Techniques}

Peel and Leakage Tests. Nonstructured PDMS was bonded to the thermoplastic films (PI, PEN, and PET) as described in section "Preparation of Microparticles". Failure of the bonding was tested by manually pulling the two substrates apart. Leakage tests were performed on completed microchannels. TB was injected through one of the PDMS inlets and the stationary liquid inside the channel was monitored for leakages using an optical microscope.

Microfilter Size Sorting

Filtration of PLGA Microparticles. For the microfilter, MPSPLGA was manually injected inside the ' $U$ ' shaped PDMS
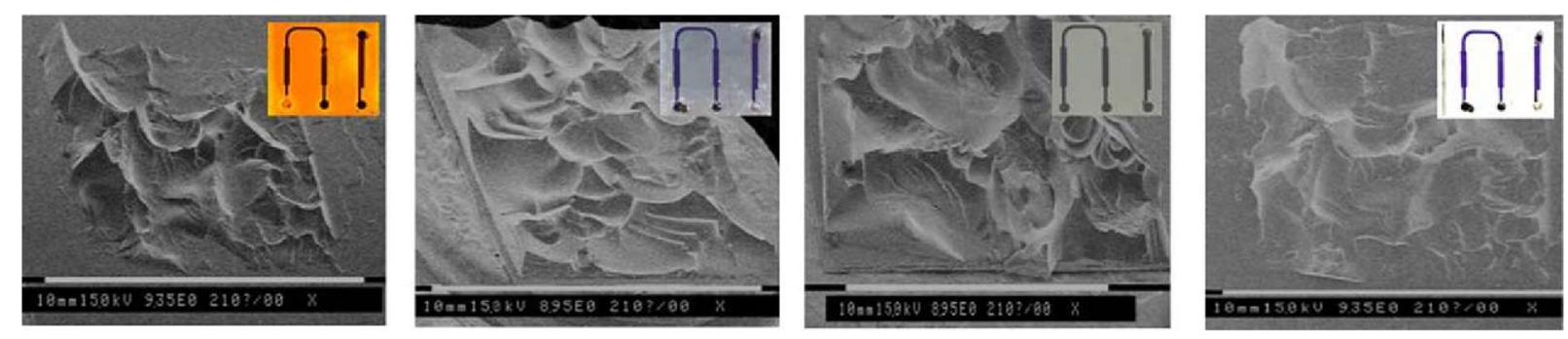

Figure 3. SEM images of a "peel test" demonstrating bonding strength on (a) PI, (b) PEN, (c) PET, and

(d) glass. Inset: Injection of TB for leakage testing. [Color figure can be viewed in the online issue,

which is available at wileyonlinelibrary.com.]

microfiltering device based on PET. The aim was to demonstrate the viability of filtering with PDMS pillar filters that are a soft material. 
Microfluidic Mixer

Mixing Performance of Polymeric Microparticles. For the micromixer, MPS-PVA was also manually injected into the PDMS device based on PET. Here, the purpose was to show the mixing performance of the size dependent MPS due to the designed micromixer. TB was also simultaneously injected from the Y-shaped inlet with premixed MPS in iron (III) nitrate. As previously reported, propanol was injected and removed prior to the introduction of the MPS. This increased the wettability of the polymers and enabled the liquids to enter into the mixing loops. ${ }^{22}$

Mixing Performance of Fluorescent Microparticles. In the same principle described in section "Mixing Performance of Polymeric Microparticles", MPS-NR were simultaneously injected with iron (III) nitrate and observed for mixing under a fluorescence microscope. The mixing performance and flow velocity along the microchannel sidewalls was observed to view the dispersion of the MPS within the microchannel intersection.

To demonstrate the applied micromixers performance against particle size, a section of the micromixer was observed with the simultaneous injection of MPS-NR and iron (III) nitrate. Here, the size-dependent MPS were observed over time to demonstrate the reduced mixing performance due to clogging within the mixing loop exits (width of $15 \mathrm{~mm}$ ).

Mixing and Size-Sorting of Microparticles

Because of the dimensions of the MPS, a PDMS microfilter and micromixer were integrated together by microfluidic tubing. Firstly, the MPS were manually injected into the ' $U$ ' shaped microfilter inlet that then flowed into the connected micromixer. The purpose was to retain and filter the larger MPS and to allow the smaller MPS $(<11 \mathrm{~mm})$ to enter into the micromixer. This will enable the sample to mix due to propulsion from the $15 \mathrm{~mm}$ mixing loop exit and reduce possible clogging. Here, MPS-NR were analyzed with iron (III) nitrate, while, non-fluorescent MPS-PVA that were premixed with iron (III) nitrate were analyzed with TB. As the MPS entered into the mixer from the connection tubing, the two dyes [iron (III) 
nitrate and $\mathrm{TB}$ ] were respectively placed at the $\mathrm{Y}$-inlet by inserting a syringe tip at the injection port. Consequently, the two liquids were brought into the micromixer by back aspiration through the exit port of the PDMS micromixer.

\section{RESULTS AND DISCUSSION}

Bonding Characterization

Peel and Leakage Tests. The strength of the APTES facilitated PDMS-polymer bond was established by the peel test. Figure 3 shows that the PDMS tended to tear above the PDMS-polymer interface, suggesting that the PDMS-polymer interface has remained intact due to the strong covalent SiAOASi bond created between the polymer and the PDMS in each case. This was confirmed by comparison to PDMS bonded to glass where a similar tearing effect within the PDMS matrix was observed [Figure 3(d)]. To compliment these findings, TB was injected into the filter systems. No leakage was observed from any of the PDMS/polymer combinations (Figure 3 inset). Consequently, it could be concluded that covalent bonding through APTES between the PDMS microfilter devices and thermoplastic polymer films was effective and strong. Characterization of the surface by contact angle measurement, surface free energy, and X-ray photoelectron spectroscopy can be found in Ref. 22.

Microfilter Application

PLGA Microparticle Filtration. The filtration of the MPS was monitored by optical microscopy (Olympus BX41M). Figure 4, 


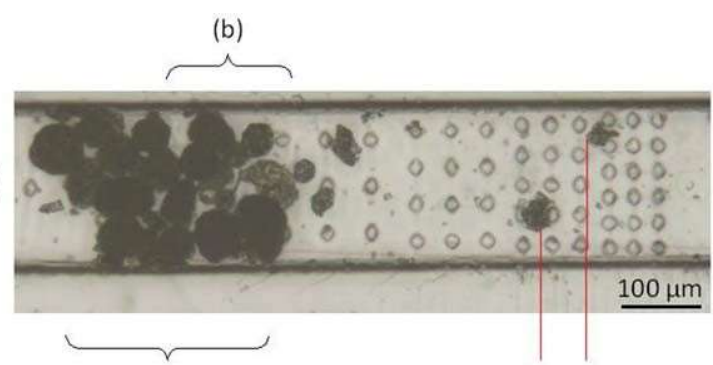

(a)

(d) $(\mathrm{e}$

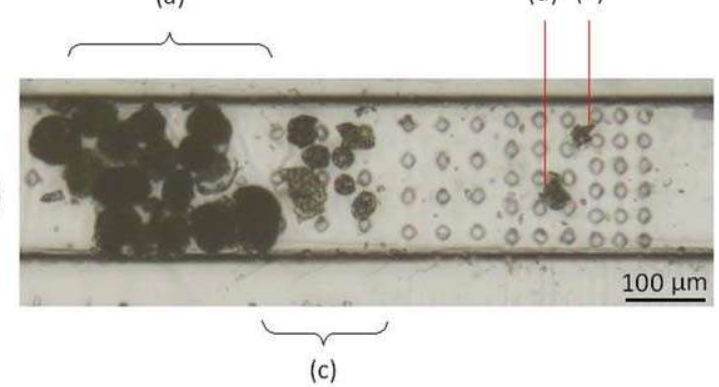

Figure 4. Optical microscope images of MPS-PLGA injected into a PDMS-PET microfiltering device containing cylindrical structures, after (1) the first injection and (2) the second injection. [Color figure can be viewed in the online issue, which is available at wileyonlinelibrary.com.]

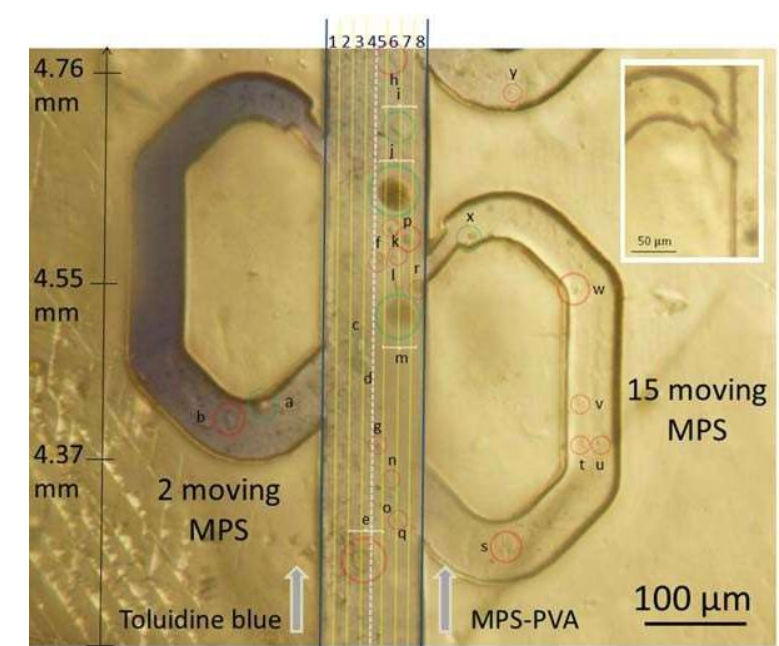

Figure 5. Optical microscope images of (a) MPS-PVA injected into a PDMS-PET micromixer device for mixing with TB at loop no. 13-14. The two liquids were introduced into the system by back aspiration with moving particles (red circle) and stationary particles (green circles). The linear intercept is significant to the half of the microchannel width with an observation of particle dispersion over the entire channel width. (Inset) Enlarged image of one large particle blocking the mixing loop exit. (The images have been 
contrast enhanced). [Color figure can be viewed in the online issue, which is available at wileyonlinelibrary.com.]

show the PDMS microfilter bonded onto APTES functionalized PET. Initially, the pressure of manual injection expedited the flow of the MPS through the microchannel due to the flexibility of the PDMS microfilters. In Figure 4, (1) we observed the first injection, followed by (2) an increased injection of MPS. In comparison of the two images, we noticed (a) a cluster of MPS that were blocked, followed by a cluster of MPS that advanced within the microchannel depending upon the filter dimensions (1b-2c). From the images, we can observe that the structures were capable of filtration, though clogging is inevitable for these types of filters. Agbangla et al. have shown the effects of latex particles with spherical structures of $4.9 \mathrm{~mm} 60.21 .^{32}$ By dead end mode filtration through micro-separators of $20 \mathrm{~mm}$, clogging was dependent upon particle concentration, flow rate, and the physical-chemical conditions of suspensions. However, in this instance the spherical dimensions of the MPS varied $(<10-50 \mathrm{~mm})$. The microchannel has a width of $200 \mathrm{~mm}$, a depth of $75 \mathrm{~mm}$, and with increasing filtration size structures based on dead end filtration then clogging of these MPS-PLGA will always occur regardless of particle concentration, flow rate, and the physical-chemical conditions of the suspension.

The particle (1d) (12.67 mm width) was stationed on the y-axis of two filters (16 mm) within Set 5. On increased injection, the particle $(2 \mathrm{~d})$ passed through the second row and remained affixed between four filters in the $\mathrm{x}$ and $\mathrm{y}$-axes of Set 5 . Here, the particle (16.72 $\mathrm{mm}$ in diameter) appears filtered from the liquid medium. Finally, at the strongest filtration point of Set $6(11 \mathrm{~mm})$, the particle $(10.72 \mathrm{~mm})$ remains outside of the first row of Set 6 (1e). With the increased injection in the image (2e), the particle (11.23 $\mathrm{mm}$ ) remains comparatively in the same position. This illustrates that the final filters (Set 6) was able to prevent a slightly larger particle from passing. This demonstrates the feasibility and application of PDMS microfilters that maintained their structural form through APTES mediated bonding method. Though 
microfilters require rigidness of the structural material (e.g., hard plastics), as shown, the filtration of a wide range of MPS sizes was capable and performed well with a soft material such as PDMS.

Micromixer Application

Microparticle Mixing. To demonstrate mixing with the aid of inks, TB and MPS-PVA [premixed with iron (III) nitrate] were both simultaneously injected into the micromixer by back aspiration. Figure 5, shows the two mixing loops significant in color to the two separate injections. Over time, the dark purple coloration within the mixing loop became similar to that in the microchannel. The coloration within the microchannel is a variation of these two colors and significant due to mixing. The liquid intersection is shown and the observed moving MPS are noted in red. As can be seen, the intersection shows more MPS that are consistent within the side of particle injection (15 moving MPS and 5 stationary MPS). These particles were randomly dispersed [i.e., close to the center and sidewalls (9 MPS) with smaller MPS noted within the mixing loop (6 MPS)]. At the intersection with TB, the MPS were central to the microchannel (MPS: 1 moving and 2 stationary) and sparse of particles within the mixing loop (MPS: 1 moving and 1 stationary). Therefore, the MPS followed a parallel laminar flow which was expected and previously observed with the mixing of two pure inks $[\mathrm{TB}$ and iron (III) $] .{ }^{22}$ Here, we observed that the MPS were nonuniform over the half of the microchannel width at the mixing loops no. 13-14 (4.27-4.76 mm). This exhibits the decreased efficiency of mixing MPS when compared to pure liquids.

Stationary and moving MPS were also observed on the surface of the PET film (Figure 5). These are significant to the hydrophobic interactions, contact forces, the roughness of the PET surface, and flow velocity. To reduce these interactions, Agbangla et al. have shown that particles dispersed in a salt solution $(\mathrm{KCl})$ are more capable in reducing the magnitude of the electrode interactions of the particles to the PDMS wall. ${ }^{32}$ A premix with a surfactant [e.g., sodium dodecyl sulphate (SDS)] may also aid in preventing particle deposits on the surface. The diameters of the MPS from Figure 5 can be found in Table I. 
With increased fluidic flow of the MPS over time, we observed clogging of the mixing loop exits. Figure 5 (inset), shows the blocking of mixing loop exit with one large particle. Because of the extent of the force pressurized onto the particle, the PDMS loop exit has contorted and expanded to accommodate the size of the particle. This demonstrates the flexibility of this soft material. However, the functionality of the micromixer at this section $(4.27-4.76 \mathrm{~mm})$ was considerably reduced at its ability to mix due to large MPS blocking the previous mixing loop exits. This was observed with a total mixing performance calculated at $13.3 \%$ of moving MPS (2/15) between 4.27 and $4.76 \mathrm{~mm}$.

Table I. Particle Size Distribution in Particular Regions of the Microchannel Consistent to the Introduction of TB and MPS-NR in Labeled Lanes of 1-8

TB MPS-PVA

\begin{tabular}{lllllllll}
\hline Mixing loop 1 & 2 & 3 & 4 & 5 & 6 & 7 & 8 & Mixing \\
& & & & & & & &
\end{tabular}
(a) 10.91
$\begin{array}{lll}\text { (c) } 5.76 & \text { (d) } 15.12\end{array}$
(f) 11.48
(h) 8.22
(p) 9.29
$\begin{array}{ll}\text { (r) } 9.88 & \text { (s) } 6.99\end{array}$

(b) 7.13

(e) 22.34

(g) 7.55

(i) 12.01

(t) 3.54

(j) 31.69

(u) 3.75
(k) 7.53
(q) 10.34
(v) 5.66

(1) 3.95

(w) 6.25

(m) 27.14

(x) 4.88

(n) 5.16

(y) 2.58 
(o) 6.38

$\begin{array}{lllllllllll}7.13-22.34 & 22.34 & 9.5262 .78 & 6.2262 .00 & 9.8260 .74 & 9.88 & 4.80 & 6\end{array}$

nb: final row 5 average particle size of moving particles (units $5 \mathrm{~mm}$ ). The moving particles are

highlighted in bold.

Figure 6 shows the simultaneous injection of fluorescent MPSNR and iron (III) nitrate. As Bhagat and Papautsky have shown, particle mixing performance is generally not efficient with Tshaped or Telsa based mixers. ${ }^{25}$ Here, we have applied the micromixer with eighteen mixing loops and observed it effectiveness. The flow velocity near to the microchannel walls is generally lower than those at the center for a typical Poiseuille flow. ${ }^{25}$ The injected MPS are inclined to redistribute within the center of the microchannel, thus moving away from the sidewalls with this principle known as shear-induced particle migration. ${ }^{33}$ However, the MPS were still observed in the microchannel and mixing loops that consisted on its initial injection and gradually to the side of iron (III) nitrate due an increase of particle concentration. For instance, Figure 6(a) shows the third and fourth loops from simultaneous injection. In a(i) there was more fluorescence on the initial side of injection for the MPS when compared to the intersection of iron (III); however, in the same image further along the microchannel the fluorescent MPS were seen to dominate the whole channel. In a(ii) an abundance of fluorescent MPS entered into the mixing loop, while an inverse for the injection of iron (III) was seen in a(iii). In the image b(i) in loop 8 minimal MPS entered into the mixing loop though an increase was observed in loop $10[\mathrm{~b}(\mathrm{ii})]$. At b(iii) the whole microchannel was saturated with fluorescent MPS. This demonstrated that the mixer was capable 

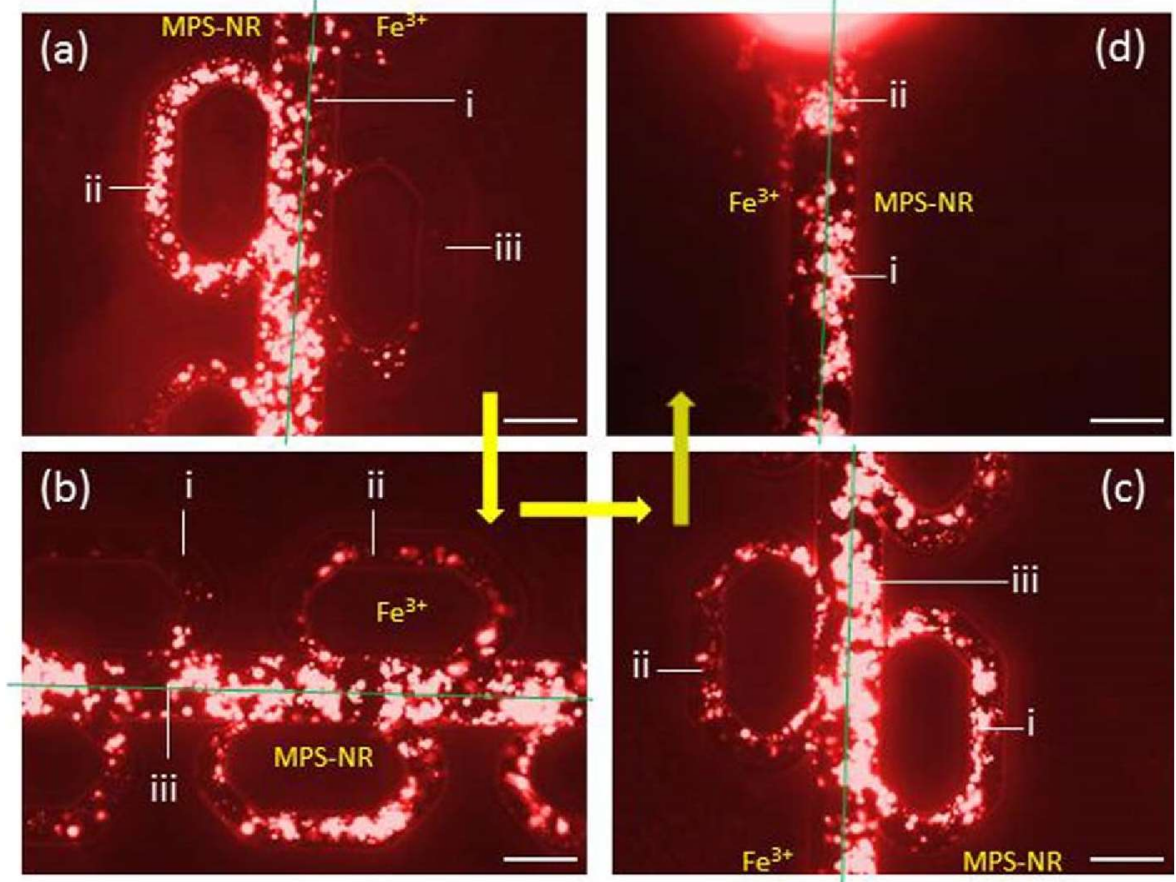

Figure 6. Micromixing by back aspiration of iron (III) nitrate and fluorescent MPS-NR within the PDMS-

PET micromixing device. The images were taken at different positions along the microchannel with (a) loops no. 3 and 4, (b) loops no. 9 and 10, (c) loops no. 13 and 14, and (d) microchannel exit (310 obj). The images have been contrast enhanced (Bar5100 mm). [Color figure can be viewed in the online issue, which is available at wileyonlinelibrary.com.]

Figure 7. Injection of TB to observe the fluidic flow from the microfilter to the micromixer, with (a) angled profile, (b) (top) top profile, and (bottom) side profile. [Color figure can be viewed in the online issue, which is available at wileyonlinelibrary.com.] 

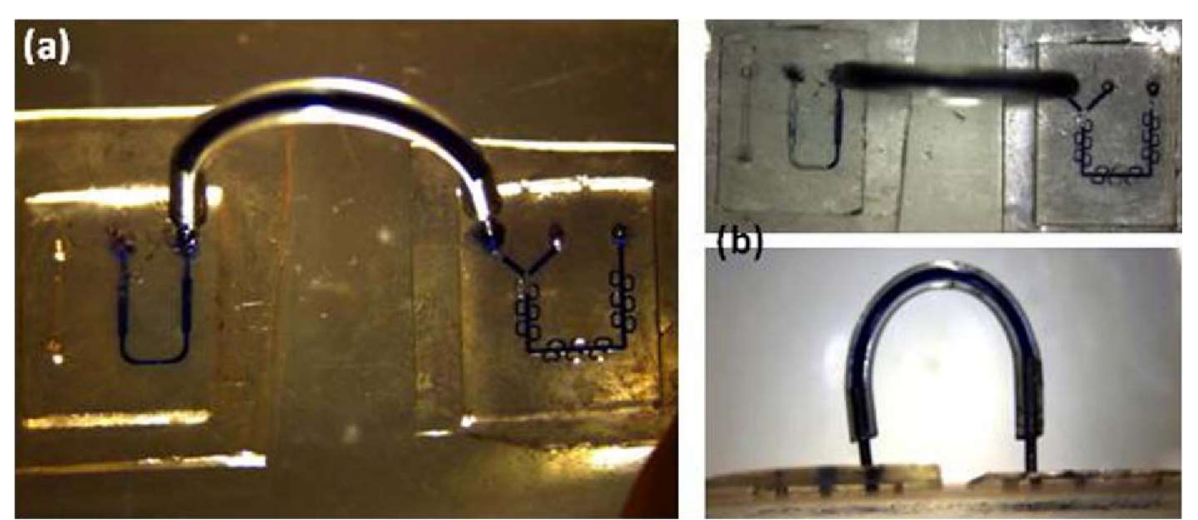

of mixing the MPS on both sides of the microchannel, with particles close to the microchannel walls. In image c(i), the fluorescence was consistent on the initial side of MPS injection, while at c(ii) an increase of particles entered into the opposing mixing loop for iron (III). At c(iii) the microchannel was saturated with fluorescent MPs though more prevalent on the side for MPS injection. In the final image (d), at d(i) more fluorescence was observed on the intersection of MPS injection and d(ii) demonstrates the clogging of the MPS at the end of the microchannel.

As the flow velocity near to the sidewalls of the microchannel is smaller than those in the center, ${ }^{25}$ here the geometry of the mixer with 18 mixing loops enables the increased particle concentration to remain near to microchannel side walls due to the fluidic flows created by the mixing loops to, first, force them to enter into the loops and second, by propulsion to return back into the microchannel for recirculation This was prevalent in Figure 6(b) where particle dispersion seemed uniform on both sides of the microchannel. For mixing of MPS, the mixing loops increased in efficiency further along the microchannel length where MPS increased in entering the mixing loops from the side of the initial injection of iron (III). However, the micromixer was capable of creating a certain amount mixing until the clogging of mixing loops $(15 \mathrm{~mm})$ had transpired. With this clogging, the MPS entering into the mixing loops were reduced throughout the whole microchannel and the fluidic flow within the mixing loops was as a direct result reduced in velocity. The particles still flowed rapidly within the microchannel, though an increased amount of fluorescence was observed on the right half of the channel [Figure 6(c,d)]. This demonstrates a nonuniform profile of the MPS on both sides of the microchannel when compared to Figure 
6(b). As there was a continuous fluidic flow, the MPS were not mixed by the mixing loops and, therefore, mixing was occurring by only molecular diffusion. Therefore, the phenomenon of shear-induced particle migration will become predominant within the microchannel as the redundant mixing loops were blocked. Overall, the varying particle sizes were capable of mixing and were drawn to the opposing mixing loops though the device was not as effective when compared to mixing pure liquids. ${ }^{22}$ Therefore, to reduce the issue of blockages due to large MPS and to ensure the MPS could mix effectively, a PDMS microfiltering device was integrated with a PDMS micromixer.

Integration of a Microfilter and Micromixer Device

Figure 7 shows the ease of connecting the microfluidic tubing to the two microfluidic devices. The flexibility of the PDMS devices and the PC tubing has enabled the inlets for the PDMS devices to support the connection through the modified syringe tips. The injection of TB was made directly into the inlet of the microfilter and the dye flowed through the ' $U$ ' shaped microchannel, through the PC tubing, and into the micromixer device. [Some small leakages were observed at the connection ports though these were resolved by sealing with a curable resin (Silicomet JS533 Red, Henkel Technologies France)].

\section{Size-Sorting and Mixing of Microparticles}

Figure $8(\mathrm{a}-\mathrm{c})$ demonstrates the direct injection of fluorescent MPS-NR into the microfilter connected to the micromixer (A couple mg of SDS was added and previously mixed with the MPS-NR to help reduce the possibility of stationary particles on the PET surface). In (a) a slow and steady flow of particles were seen within the first barrier (rectangular structures) and those particles small enough were capable of passing through the post structures. In (b) and with increased injection due to the pressure required for the fluidic flow to pass through the connector, increased particles were seen within the microchannel. However, by (c), the microfiltering device was completely saturated with MPS-NR and the device was clogged. In Figure 8(d), some particles (n511) passed through the connector with manual and simultaneous 
injection of iron (III) through the other inlet of the micromixer. It was observed that more particles remained on the side of introduction for the particles that were also larger in diameter [7 MPS ( $\mathrm{x}$ $59.4862 .59 \mathrm{~mm}$ ) as compared to 4 MPS (x56.92 $63.43 \mathrm{~mm})$ ]. At this section, the particles did not pass through the mixing loops. However, these particles were small enough to pass through the mixing loops in the micromixer and unlike those previously shown (Figure 6). Therefore, the mixing due to the total number of particles (11 MPS) observed in the microchannel
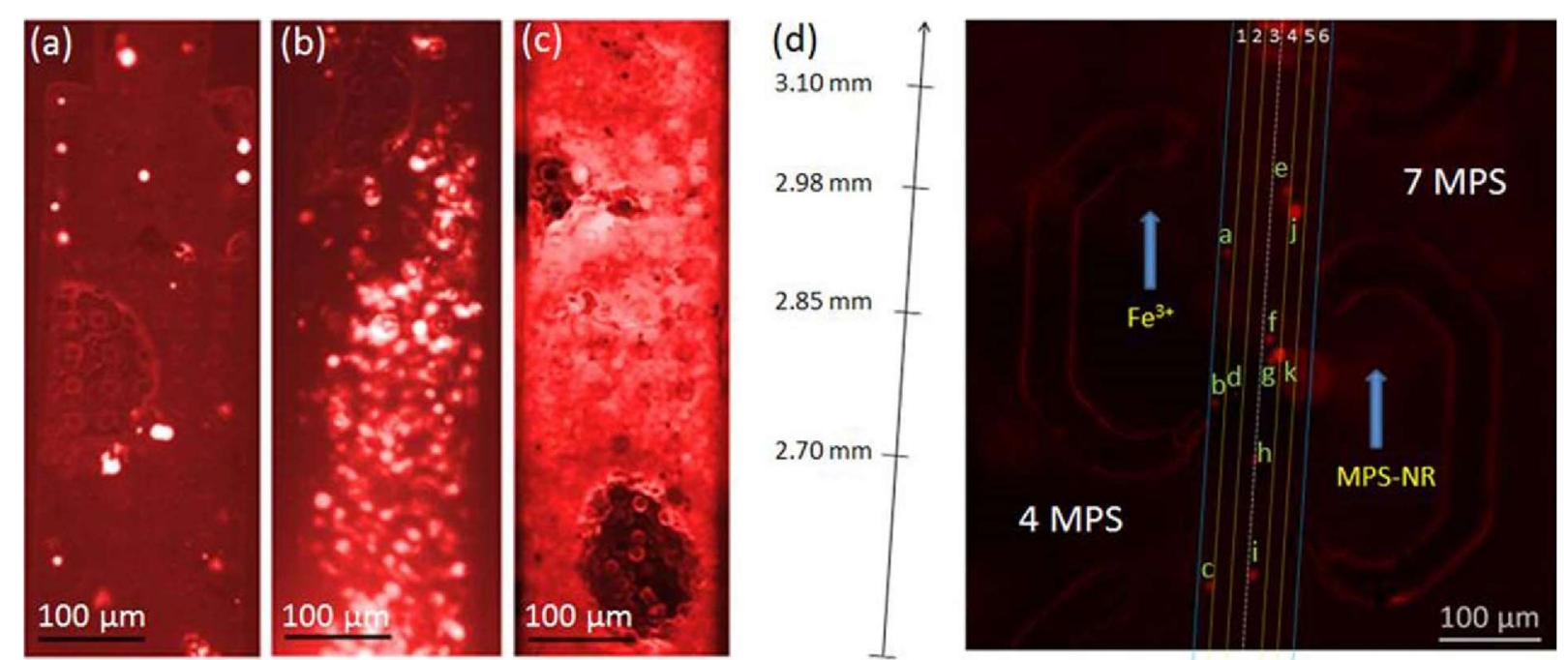

Figure 8. Injection of labeled MPS-NR through the microfiltering device with (a) entry and passage of small particles, (b) increased injection, and (c) complete saturation of MPS within the microchannel. (d) Fluorescent image of the MPS-NR entering into the mixer with the simultaneous injection of iron (III). Image taken at loops 9-10. [Color figure can be viewed in the online issue, which is available at wileyonlinelibrary.com.] for the intersection was calculated at 36.4\% [iron (III)] and 63.6\% (MPS-NR).

In Table II, we can observe the distribution of the particle sizes in accordance to Figure 8(d). In the sixth column for the initial introduction of MPS-NR there were no particles observed at the microchannel wall. In the fifth column, a large particle has passed through the $11 \mathrm{~mm}$ size sorting structures [x513.07 60.15 mm (n 52)]. This was due to the soft and flexible nature of PDMS; however, only two large particles had 
passed through the filtering system and remained within the middle lane for MPS-NR. (It must be noted that the diameters of these MPS may be larger due to the amount of fluorescence that is being emitted). At the intercept of iron (III) with MPSNR, in lane 4 a multitude of MPS were observed and significant to shear-induced particle migration. These particles had successfully passed through the size sorting microfilters at $\mathrm{x} 58.0561 .03 \mathrm{~mm}(\mathrm{n} 55)$. At the intercept in lane 3, there were no observed MPS. In lane 2, a small particle at $2.76 \mathrm{~mm}$ was seen and demonstrates the migration from the side of MPS-NR to iron (III). This was improved by further MPS at lane 1 near to the sidewall for the introduction of iron (III). These particles [x58.31 $62.48 \mathrm{~mm}$ (n53)] were similar to the diameters observed in lane 4 corresponding to the intercept between iron (III) and MPS-NR. This demonstrates the nonuniform mixing of particles at loops $9-10$ with a mixing performance of $57 \%(4 / 7)$ at $2.6-3.10 \mathrm{~mm}$.

For nonfluorescing MPS-PVA, in Figure 9(a) we observed a multitude of MPS filtered within the PDMS structures and those small enough passed through the microfluidic system, into the connector, and then into the micromixer at loops 9-10 (2.6-3.10 mm). (A couple mg of SDS was added and previously mixed with the MPS-PVA). In Figure 9(b), we observed small MPS that were nonfiltered and these entered into the mixer that shows a success of size sorting when compared to Figure 5. Here, we observed more MPS within the mixing loop from the side of introduction from the microfilter (6 MPS). For the injection of TB, we observed some MPS in the mixing loop (4 MPS) and this was an improvement when compared to Figure 5. This was due to the introduction of smaller MPS that were not blocking the mixing loop exits. In the microchannel itself,

Table II. Particle Size Distribution in Particular Regions of the Microchannel Consistent to the Introduction of Iron (III) Nitrate and MPS-NR in Labeled Lanes of 1-6

Iron (III) nitrate $\quad$ MPS-NR 
2

3

4

5

(a) $8.33 \mathrm{~mm}$

(d) $2.76 \mathrm{~mm}$

(e) $9.39 \mathrm{~mm}$

(j) $13.17 \mathrm{~mm}$

(b) $5.82 \mathrm{~mm}$

(f) $7.42 \mathrm{~mm}$

(k) $12.96 \mathrm{~mm}$

(c) $10.77 \mathrm{~mm}$

(g) $6.70 \mathrm{~mm}$

(h) $8.28 \mathrm{~mm}$

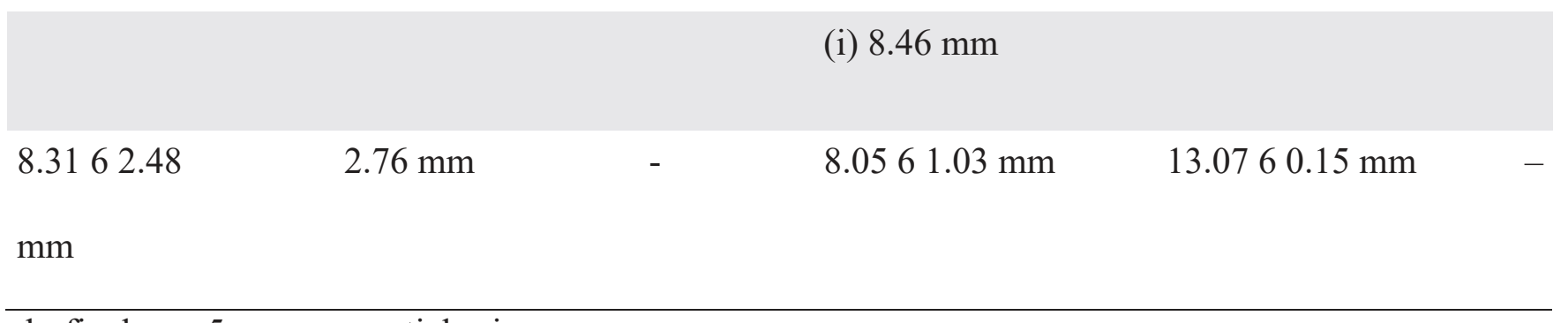

nb: final row 5 average particle size.
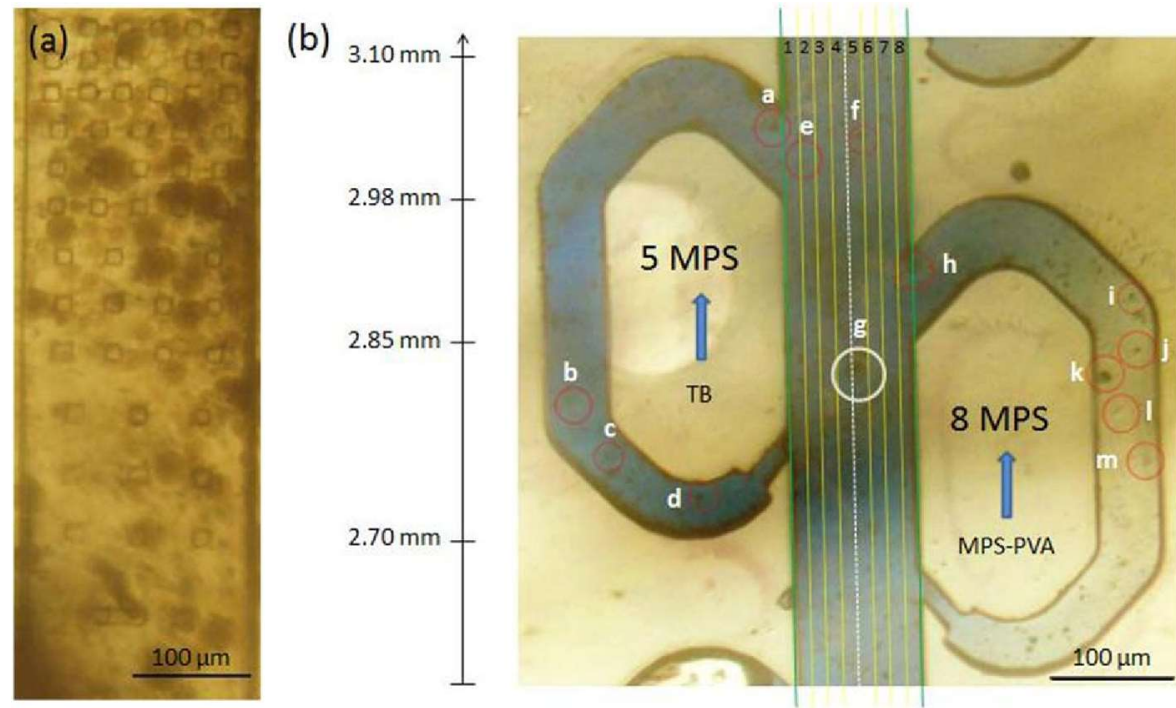

Figure 9. (a) Filtration by optical microscopy of MPS-PVA premixed with iron (III) nitrate (310 obj). (b) MPS-PVA premixed with iron (III) and with the simultaneous injection of TB. One large particle that bypassed the filters is highlighted in white. Image taken at loops 9-10. Both images have been contrast enhanced. [Color figure can be viewed in the online issue, which is available at wileyonlinelibrary.com.] 
only one particle was observed in lane 2 for TB and two MPS in lane 5 that were near to the intercept of TB with MPS-PVA. As there were more MPS in the mixing loops than in the microchannel, this can signify that filtration followed by mixing with small enough particles had enabled an improved efficiency of the MPS entering into the mixing loops for mixing. It is well-known that particles tend to follow a more central trajectory in the microchannel though there are multiple factors that can affect particle migration. ${ }^{34,35}$ Because of their size and mass, large MPS were prevented from entering into the mixing loops and followed the fluidic streamlines. However, the difficulty of mixing MPS when compared to pure liquids can be observed with a nonuniform number of MPS over the half of the microchannel width. The mixing due to the total number of particles (13 MPS) noted in the microchannel for the intersection was calculated at $38.5 \%$ (TB) and $61.5 \%$ (MPS-PVA). This makes evident the nonuniform mixing of particles at loops 9-10, though an improvement was observed when the MPS were smaller and when the mixing loops were functional (nonblocked). Therefore, a $62.5 \%(5 / 8)$ mixing performance from the intercept of TB and MPS-PVA was calculated at a microchannel length of 2.6-3.10 mm. 
Table III. Particle Size Distribution in Particular Regions of the Microchannel Consistent to the Introduction of Iron (III) Nitrate and MPS-PVA in Labeled Lanes of 1-8

\begin{tabular}{|c|c|c|c|c|c|c|c|c|c|}
\hline \multicolumn{5}{|c|}{ TB } & \multicolumn{5}{|c|}{ MPS-PVA } \\
\hline Mixing loop & 1 & 2 & 3 & 4 & 5 & 6 & 7 & 8 & Mixing loop \\
\hline (a) $9.75 \mathrm{~mm}$ & & (e) $7.31 \mathrm{~mm}$ & & & (f) $6.09 \mathrm{~mm}$ & & & & (h) $9.14 \mathrm{~mm}$ \\
\hline (b) $7.92 \mathrm{~mm}$ & & & & & (g) $15.24 \mathrm{~mm}$ & & & & (i) $6.09 \mathrm{~mm}$ \\
\hline (c) $6.70 \mathrm{~mm}$ & & & & & & & & & (j) $4.27 \mathrm{~mm}$ \\
\hline \multirow[t]{3}{*}{ (d) $7.31 \mathrm{~mm}$} & & & & & & & & & (k) $2.44 \mathrm{~mm}$ \\
\hline & & & & & & & & & (1) $5.49 \mathrm{~mm}$ \\
\hline & & & & & & & & & $\begin{array}{l}\text { (m) } 6.70 \\
\mathrm{~mm}\end{array}$ \\
\hline $7.9261 .32 \mathrm{~mm}$ & - & $7.31 \mathrm{~mm}$ & - & - & $10.6766 .47 \mathrm{~mm}$ & - & - & - & $\begin{array}{lll}5.69 & 6 & 2.27 \\
\mathrm{~mm} & & \end{array}$ \\
\hline
\end{tabular}


nb: final row 5 average particle size.

In Table III, we can observe the distribution of the particle sizes in accordance to Figure 9(b). Within the microchannel there were three observable MPS with the largest measured at $15.24 \mathrm{~mm}(\mathrm{e})$. The mixing loops for the introduction of MPS-PVA were smaller [x55.6962.27 mm (n56)] than those observed with the addition of the mixing liquid, TB [x57.9261.32 mm (n54)]. This demonstrates that the previous size sorting followed by mixing has enabled small MPS to overcome migration from the centre of the microchannel and enter within the mixing loop with TB from the opposing side of the initial injection of MPS-PVA. This was aided by the minimization of the mixing loop exit blockages, thus, allowing the MPS to be influenced by the forces generated by the streams created at the mixing loop exits. In comparison of Figure 5 with 9 (b), we can observe that smaller particles tended to remain within the mixing loops of the initial side for MPS-PVA with diameters between 5 and $6 \mathrm{~mm}$ [4.8061.75 mm (n 56) and $5.6962 .27 \mathrm{~mm}$ (n56), respectively]. Because of their smaller diameters and weight, it can be assumed that the fluidic flows reduced the amount of MPS from migrating to the opposing intercept of the microchannel. MPS that migrated from the intercept and into the opposing mixing loops for TB were larger at 7-8 $\mathrm{mm}$ [Table I: $7.13 \mathrm{~mm}$ (n 51) and Table III: $7.9261 .32 \mathrm{~mm}$ (n54)]. Therefore, these larger MPS were capable of overcoming the fluidic forces created at the entrance of the mixing loops (50 $\mathrm{mm}$ diameter) for MPS-PVA that could then migrate to the centre of the microchannel and then be drawn towards the entrance of the mixing loops for TB. This suggests that the applied micromixer performance is dependent upon particle size. However, due to the size of the MPS (Figure 5), we observed that the micromixers performance 
$(13.3 \%$ in $5 \mathrm{~mm})$ was dramatically reduced when large MPS entered into the mixer as this created blockages of the mixing loop exit. Upon filtration of the MPS to $<11 \mathrm{~mm}$, the mixing loops were unaffected and the mixing performance increased to $62.5 \%$ in $3 \mathrm{~mm}$.

Overall, for Figure 9(b), the microchannel is more consistent of the ink (TB) due to the ease of introduction from the open Yinlet. Generally, the introduction of the ink was facile when compared to the MPS due to the build-up of pressure generated from the clogging of the microfilters. By back aspiration through the exit port, the ink entered more rapidly though particles were still observed within the blue colored microchannel and mixing loop [Figure 9(b)]. The drawback of applying flexible PDMS microstructured filters can be observed with one large particle observed within the white circle. Here, the particle had bypassed the filters and entered into the micromixer. Compared to the introduction of MPS within the singular microfiltering device, where the introduction of the particles was made by bask aspiration. Here, the MPS were directly injected into the injection port of the PDMS microfiltering system. Because of the internal pressure created to ensure the MPS passed through the connection, an increased amount of pressure was applied to force the MPS into the micromixer. As the PDMS is flexible and with this increased pressure to drive the liquid over to the other microfluidic device, the large particle $(15.24 \mathrm{~mm}$ diameter) was observed within the microchannel. To reduce this phenomenon, the PDMS at a ratio of 10 $: 1(\mathrm{w} / \mathrm{w})$ can be reduced to a ratio of $5: 1(\mathrm{w} / \mathrm{w})$ as this will improve the stiffness and rigidity of the posts. The Young's modulus is E5580 $\mathrm{kPa}[10: 1(\mathrm{w} / \mathrm{w})]$ and E $51000 \mathrm{kPa}[5: 1(\mathrm{w} / \mathrm{w})]$ of the different curing agent mixtures. ${ }^{36}$ Alternatively, hard PDMS (h-PDMS) has a tensile modulus that is 4.5 times that of standard PDMS ${ }^{37}$ This will provide the structured microfilters to withstand the fluidic forces and MPS from passing through the microchannel and provide an alternative to hard thermoplastic films developed by expensive machinery such as hot embossing lithography (HEL) and nanoimprint lithography (NIL). 


\section{CONCLUSIONS}

In this work, the fabrication of PDMS microfilters has been successfully achieved by a double casting process using gold as an antiadhesive material for replica molding using two PDMS molds. These PDMS filters have been chemically bonded to PI, PEN, and PET substrates using APTES chemical functionalization. These preliminary results show the successful filtration of MPS-PLGA in PDMS pillar posts followed by MPS mixing in passive PDMS micromixers based on flexible thermoplastic films. The feasibility and testing for the integration of multipurpose microfluidics using polymeric microfilters and micromixers has demonstrated the ease of merging the two microfluidic applications together with achievable filtration and mixing of MPS. Without prior filtration, the micromixer tended to clog due to the influx of large MPS, while particle dispersion was observed as nonuniform. This demonstrated the difficulty of homogeneously mixing particles from two solutions at the Yinlet. After size-sorting and mixing, clogging was greatly reduced due to the critical number of MPS that flowed from the microfilter and into the micromixer. This was assisted with the addition of the secondary ink that was injected at the other Y-inlet of the micromixer. These nonfiltered MPS also appeared more capable of entering into the mixing loops after they entered into the micromixer due to their smaller diameters $(<11 \mathrm{~mm})$. However, mixing of the MPS was also nonuniform on moving across the microchannel width. This demonstrates that the designed eighteen mixing loops have some effect on mixing particles $(7-8>\mathrm{mm})$ though the mixing performance of the device was calculated at $62.5 \%$ in $3 \mathrm{~mm}$. Finally, future work will determine the tensile strength by load-displacement curves to evaluate the bonding strength, while the ultimate concept for these devices will be used for the integration within mTAS applications for mixing and filtration of biological samples, for example, whole blood samples. 


\section{ACKNOWLEDGMENTS}

The authors would like to thank Dr Francois Bessueille (Institut de Sciences Analytiques (ISA), Universite de Lyon1, France) and Dr Chris Mills (Advanced Technology Institute, University of Surrey, UK) for their advice during the completion of this work. We acknowledge the funding through the Ministe're de L'enseignement Superieur et de la Recherche (MESR), the European Communities Seventh Framework Programmes; SensorART (FP7/2007-2013) under the grant agreement No. 248763 and SEA-on-a-CHIP (FP7OCEAN-2013) under grant agreement No. 614168, FP7-PEOPLE2012-IRSES under the grant agreement No. 318053 (SMARTCANCERSENS), SPS (NUKP.SFPP984173) NATO project, and by the Spanish Government through the MINAHE 3 project MEC-

TEC2008-06883-CO3-01, and it reflects only the authors' views.

\section{REFERENCES}

1. Niskanen, A. J.; Ylinen-Hinkka, T.; Kulmala, S.; Frannssila,

S. Sens. Actuators B. 2011, 152, 56.

2. Qiu, J.; Zhou, Y.; Chen, H.; Lin, J.-M. Talanta 2009, 79,

787.

3. Held, M.; Lee, A. P.; Edwards, C.; Nicolau, D. V. Microelectron. Eng. 2010, 87, 786.

4. Kuo, T. C.; Cannon, D. M., Jr.; Chen, Y., Tulock J. J.Shannon; M. A.; Sweedler J. V.; Bohn; P. W Anal. Chem 2003, 75, 1861

5. Mills, C. A.; Navarro, M.; Engel, E.; Martinez, E.; Ginebra,M. P.; Planell, J.; Errachid, A.; Samitier, J. J. Biomed. Mater. Res. 2006, 76, 781

6. Mills, C. A.; Escarre, J.; Engel, E.; Martinez, E.; Errachid, A.;Bertomeu, J.; Andreu, J.; Planell, J. A.; Samitier, J. Nanotechnology 2005, 16, 369 
7. Mills, C. A.; Fernandez, J. G.; Errachid, A.; Samitier, J.Microelectron. Eng. 2008, 85, 1897.

8. Mills, C. A.; Martınez, E.; Errachid, A.; Gomila, G.; Samso,

A.; Samitier, J. Contrib. Sci. 2005, 3, 47.

9. Mills, C. A.; Martinez, E.; Bessueille, F.; Villanueva, G.;Bausells, J.; Samitier, J.; Errachid, A. Microelectron. Eng. 2005, 78, 695.

10. Tennico, Y. H.; Koesdjojo, M. T.; Kondo, S.; Mandrell, D. T.;Remcho, V. T. Sens. Actuators B 2010, $143,799$.

11. Hwang, K.-Y.; Kim, J.-H.; Suh, K.-Y.; Ko, J. S.; Huh, N.Sens. Actuators B 2011, 155, 422.

12. Aran, K.; Sasso, L. A.; Kamdar, N.; Zahn, J. D. Lab Chip 2010, 10, 548.

13. Tang, L.; Lee, N. Y. Lab Chip 2010, 10, 1274.

14. Sunkara, V.; Park, D.-K.; Hwang, H.; Chantiwas, R.; Soper,

S. A.; Cho, Y.-K. Lab Chip 2010, 11, 962.

15. Goodman, S. L.; Sims, P. A.; Albrecht, R. M. Biomaterials 1996, 17, 2087.

16. Xia, Y.; Whitesides, G. M. Angew. Chem. Int. Ed. 1998, 37, 550.

17. Xia, Y.; Venkateswaran, N.; Qin, D.; Tien, J.; Whitesides, G.M. Langmuir 1998, 14, 363.

18. Li, C.; Wilkes, G. L. J. Inorg. Organomet. 1998, 8, 33.

19. Hu, B.; Siddiqui, J. A.; Ottenbrite, M. Macromol. Chem. Phys. 2002, 203, 1631.

20. Becker, H.; Locascio, L. E. Talanta 2002, 56, 267.

21. Gu, Y.; Miki, N. Microelectron. Eng. 2007, 17, 2308

22. Lee, M.; Lopez-Martinez, M. J.; Baraket, A.; Zine, N.; Esteve, J.; Plaza, J. A.; Jaffrezic-Renault, N.; Errachid, A. J. Polym. Sci. Part A: Polym. Chem. 2012, 51, 59.

23. Choi, J.-W.; Oh, K. W.; Han, A.; Okulan, N.; 
Wijayawardhana, C. A.; Lannes, C.; Bhansali, S.; Schlueter, K. T.; Heineman, W. R.; Halsall, H. B.; Nevin, J. H.; Helmicki, A. J.; Henderson, H. T.; Ahn, C. H. Biomed. Microdevices 2001, 3, 191.

24. Yuen, P. K.; Kricka, L. J.; Fortina, P.; Panaro, N. J.; Sakazume, T.; Wilding, P. Genome Res. 2001, 11, 405.

25. Bhagat, A.; Papautsky, I. J. Micromech. Microeng. 2008, 18, 085005.

26. Lopez-Martinez, M. J.; Campo, E. M.; Caballero, D.; Fernandez, E.; Errachid, A.; Esteve, J.; Plaza, J.

A. J. Micromech. Microeng. 2009, 19, 105013.

27. Lopez-Martinez, M. J. PhD Dissertation, Universitat Autonoma de Barcelona,2008, 257-267.

28. Caballero, D.; Samitier, J.; Bausells, J.; Errachid, A. Small 2009, 5, 1531.

29. Cotte, S.; Baraket, A.; Bessueille, F.; Gout, S.; Yaakoubi, N.;Jaffrezic-Renault, N.; Leonard, D.;

Errachid, A. Positive Microcontact Printing using Wet Palladium Deposition. Proceedings of the Biosensors 2010 World Congress, Glasgow, 26- 28 May 2010.

30. Kalaji, N.; Sheibat-Othman, N.; Saadaoui, H.; Elaissari, A.;Fessi, H. e-Polymers 2009, $10,1$.

31. Ayoub, M.; Ahmed, N.; Kalaji, N.; Charcosset, C.; Magdy, A.;Fessi, H.; Elaissari, A. J. Biomed. Nanotechnol. 2011, 7, 255.

32. Agbangla, G. C.; Climent, E.; Bacchin, P. Sep. Purif. Technol. 2012, 101, 42.

33. Frank, M.; Anderson, D.; Weeks, E. R.; Morris, J. F. J. Fluid Mech. 2003, 493, 363.

34. Ookawara, S.; Street, D.; Ogawa, K. Chem. Eng. Sci. 2006, 61, 3714.

35. Semwogerere, D.; Weeks, E. R. Phys. Fluids 2008, 20,

043306

36. Park, J. Y.; Yoo, S. J.; Lee, E.-J.; Lee, D. H.; Kim, J. Y.; Lee,

S.-H. Bio. Chip J. 2010, 4, 230.

37. Choi, K. M.; Rogers, J. A. J. Am. Chem. Soc. 2003, 125 , 
4060. 